OPEN ACCESS

Edited by:

Roberto Monastero, Università degli Studi di Palermo, Italy

Reviewed by: Panteleimon Giannakopoulos, Université de Genève, Switzerland Zhi Yang,

Shanghai Key Laboratory of Psychotic Disorders, China

*Correspondence: Peipeng Liang ppliang@cnu.edu.cn Xiaolan Fu

fuxl@psych.ac.cn

Received: 23 April 2018 Accepted: 10 December 2018 Published: 10 January 2019

Citation: Jia X, Li Y, Li K, Liang P and Fu X (2019) Precuneus Dysfunction in

Parkinson's Disease With Mild Cognitive Impairment. Front. Aging Neurosci. 10:427. doi: 10.3389/fnagi.2018.00427

\section{Precuneus Dysfunction in Parkinson's Disease With Mild Cognitive Impairment}

\author{
Xiuqin Jia ${ }^{1,2}$, Ying $\mathrm{Li}^{3}$, Kuncheng $\mathrm{Li}^{3,4}$, Peipeng Liang ${ }^{5 *}$ and Xiaolan $\mathrm{Fu}{ }^{1,6 *}$ \\ ${ }^{1}$ State Key Laboratory of Brain and Cognitive Science, Institute of Psychology, Chinese Academy of Sciences, Beijing, China, \\ ${ }^{2}$ Department of Radiology, Beijing Chaoyang Hospital, Capital Medical University, Beijing, China, ${ }^{3}$ Department of Radiology, \\ Anzhen Hospital, Capital Medical University, Beijing, China, ${ }^{4}$ Beijing Key Lab of MRI and Brain Informatics, Beijing, China, \\ ${ }^{5}$ School of Psychology, Capital Normal University, Beijing, China, ${ }^{6}$ Department of Psychology, University of the Chinese \\ Academy of Sciences, Beijing, China
}

Background: Mild cognitive impairment (MCl) frequently occurs in Parkinson's disease (PD). Neurovascular changes interact with neurodegenerative processes in PD. However, the deficits of cerebral blood flow (CBF) perfusion and the associated functional connectivity $(\mathrm{FC})$ in $\mathrm{PD}$ patients with $\mathrm{MCl}(\mathrm{PD}-\mathrm{MCl})$ remain unclear.

Purpose: This study aimed to explore the specific neurovascular perfusion alterations in PD-MCl compared to PD with normal cognition (PD-NC) and healthy controls (HCs), and to further examine the resultant whole brain FC changes in the abnormal perfusion regions.

Methods: Relative CBF (rCBF) was calculated using arterial spin labeling (ASL) in 54 patients with PD (27 patients with PD-NC and 27 patients with PD-MCl) and $25 \mathrm{HCs}$ matched for age and gender ratio, who also underwent the structural MRI, resting-state functional MRI (rs-fMRI) and neuropsychological examinations. The gray matter (GM) changes in PD patients were analyzed using voxel-based morphometry (VBM). The alterations in rCBF perfusion and FC among groups were then analyzed respectively. Additionally, correlations between these alterations and neuropsychological performances were further examined.

Results: Compared to HC, left caudate atrophy was detected in patients with PD. In comparison to both PD-NC and $\mathrm{HC}$, patients with PD-MCl specifically exhibited hypoperfusion in the parietal memory network (PMN) in the precuneus (PCu) and decreased PCu-FC in the right striatum. Moreover, PCu perfusion and PCu-FC strengths in the right striatum were positively associated with memory performance in PD-MCl.

Conclusions: These findings suggest that the posterior PMN dysfunction underlies memory deficits in PD-MCl.

Keywords: Parkinson's disease with mild cognitive impairment, parietal memory network, arterial spin labeling (ASL), functional connectivity (FC), precuneus 


\section{INTRODUCTION}

Cognitive decline occurs commonly and frequently in patients with Parkinson's disease (PD). Mild cognitive impairment in PD patients (PD-MCI) appears to elevate the risk for developing dementia (Janvin et al., 2006; Kehagia et al., 2010) and may even precede motor symptoms (Muslimovic et al., 2005; Aarsland et al., 2009). Examining brain function in individuals with PD-MCI may improve identification of potential markers of PD with dementia prior to the onset of significant clinical symptoms. Neurovascular dysfunction may contribute to cognitive decline in PD (Melzer et al., 2011; Fernández-Seara et al., 2012). However, little is known about the functional abnormalities that underpin the manifestation of $\mathrm{MCI}$ in this disease compared to $\mathrm{PD}$ with normal cognition (PD-NC) and healthy controls (HCs).

Arterial spin labeling (ASL) enables the quantitative measurement of cerebral blood flow (CBF; mL blood/100 g tissue/min) by using endogenous arterial blood water magnetization as a noninvasive tracer (Detre et al., 1992; Williams et al., 1992; Wolf and Detre, 2007). CBF perfusionassociated exchange of oxygen and nutrients occurring at the capillary level is considered as a close indicator of neuronal activity and glucose metabolism (Musiek et al., 2012). Abnormal CBF perfusions in the posterior cortex in PD (Melzer et al., 2011; Fernández-Seara et al., 2012) largely overlap with those reported for fluorodeoxyglucose PET metabolic deficits in this disease (Ma et al., 2007, 2010; Hosokai et al., 2009; Teune et al., 2014), and thus indicate that CBF perfusion could work as a biomarker of the neurodegenerative process in $\mathrm{PD}$.

Of note, there are few CBF perfusion studies in PD-MCI. In those non-demented PD studies, the patients with PD-MCI are not differentiated from PD-NC (Fernández-Seara et al., 2012; AI-Bachari et al., 2014), which is mainly due to the lack of comprehensive definition of PD-MCI before its release by the Movement Disorder Society Task Force (Litvan et al., 2012). However, the CBF findings in PD-MCI are inconsistent and remain inconclusive. For example, posterior parieto-occipital cortex hypoperfusion in PD-MCI has been highlighted in a SPECT study in comparison to controls (Nobili et al., 2009). In another study, CBF perfusion has been investigated in $\mathrm{PD}$ with the entire spectrum of cognitive status (including NC, MCI, and dementia) using ASL, which therefore fails to identify the alteration in CBF perfusion specific to PD-MCI (Melzer et al., 2011). On the other hand, studies with non-demented PD (combined with part of patients with MCI) exhibited either subcortical hypoperfusion and widespread cortical hypoperfusion in the posterior parieto-occipital cortex, precuneus $(\mathrm{PCu}) /$ cuneus, and frontal cortex coupled with some of them being atrophied (Fernández-Seara et al., 2012), or failed to find any group difference in non-demented PD compared to HC (AI-Bachari et al., 2014). These inconsistencies may result from the heterogeneity in PD-MCI samples and the confounding effect (e.g., ON medication or OFF medication) on results between group comparisons. Accordingly, the specific perfusion alteration in well-defined PD-MCI has not been fully described and remains unclear in comparison with PD-NC and HCs. On the basis of previous studies (Nobili et al., 2009;
Fernández-Seara et al., 2012), it was hypothesized that the PD-MCI might exhibit hypoperfusion in the parietal-occipital network.

Moreover, it has been previously proposed that the alteration in neurovascular perfusion significantly affects the blood oxygenation level-dependent (BOLD) signal (D'Esposito et al., 2003) and is associated with functional dysconnectivity (Yoshiura et al., 2009). Low-frequency BOLD signal in restingstate functional MRI (rs-fMRI) provides a sensitive measure of brain activity. Several previous resting-state FC (rs-FC) studies have addressed functional alterations in PD-MCI. These studies have demonstrated that PD-MCI exhibits FC alterations mainly in the dorsal attention network (DAN), default mode network (DMN) and fronto-parietal network (FPN) in PD-MCI (Amboni et al., 2015; Baggio et al., 2015; Chen et al., 2017; Bezdicek et al., 2018). However, how the altered neurovascular perfusion interacts with the disrupted FC remains still unclear. To capture the subtle neural changes occurring in PD-MCI, seed-to-voxel FC, an approach to assess functional integrity among structurally segregated brain regions, was further conducted based on abnormal CBF perfusion regions in PD-MCI.

Hence, the present study first aimed to examine the alterations of $\mathrm{CBF}$ perfusion specific to $\mathrm{PD}-\mathrm{MCI}$ compared to PD-NC and HC. Based on previous findings that CBF alterations occur gradually in PD patients with cognitive progression (Melzer et al., 2011) and the posterior cortical hypoperfusion is related to cognitive decline in non-demented PD (Syrimi et al., 2017), it was thus hypothesized that PD-MCI might exhibit abnormal CBF perfusion in posterior parietal cortical regions. Second, the present study aimed to explore the changes of neural activity by $\mathrm{FC}$ in the altered perfusion regions in PD-MCI compared to PD-NC and HC. It was further hypothesized that functional dysconnectivity may appear in $\mathrm{PD}-\mathrm{MCI}$ in regions with altered CBF perfusion in this disease. Finally, the present study aimed to reveal how these brain degenerations associate with neuropsychological deficits in PD-MCI.

\section{MATERIALS AND METHODS}

\section{Subjects}

In the present study, 60 patients with PD (30 for PD-NC and 30 for PD-MCI) who met the UK Bank criteria for the diagnosis of PD (Hughes et al., 1992) were recruited in the present study. All PD participants were examined in their OFF medication state, that is, at least after a 12-h withdrawal of anti-Parkinson medication. The HC group comprised 30 volunteers who were matched for age and gender ratio. Six patients and five healthy volunteers were excluded from the study due to motion artifacts and low quality of MRI data. At last, 27 PD-NC (15 males, $63.11 \pm 9.27$ years old), 27 PD-MCI (16 males, $62.59 \pm 6.61$ years old), and $25 \mathrm{HCs}$ (11 males, $59.44 \pm 5.77$ years old) were included in this study. None of the participants suffer from depression, determined with the 17-item Hamilton Depression Rating Scale (HAMD-17; Hamilton, 1960). Patients with MCI were defined by Level II of the Movement Disorder Society Task Force (Litvan et al., 2012). The study was approved by the 
Research Ethics Committee of Xuanwu Hospital, Capital Medical University and written informed consent was obtained from each participant.

\section{Diagnostic Criteria and Neuropsychological Assessment}

For the clinical characteristics, disease stage was scored using the $\mathrm{H} \& \mathrm{Y}$ state score and disease severity was captured by the UPDRS part III. The neuropsychological tests that included two tests within each of the five cognitive domains were as follows: (i) Trait Making Test and Digit Span Test within attention and working memory domain; (ii) Verbal Fluency Test and Clock Drawing Test within executive domain; (iii) Wechsler Adult Intelligence Scale-IV (WAIS-IV) and Boston Naming Test within language domain; (iv) Rey's Auditory Verbal Learning Test (AVLT) and Logical Memory subset within memory domain; and (v) Benton's Judgment of Line Orientation (JOLO) and Clock copying within visual spatial function domain.

On the basis of comprehensive neuropsychological tests, patients with PD were classified as cognitively normal and with MCI. The cognitive impairment in PD was defined based on the detailed cognitive battery: expected $\mathrm{z}$-scores for each test and each subject were calculated based on a multiple regression analysis performed in the $\mathrm{HC}$ group adjusted for age, gender, and education (Aarsland et al., 2009). A subject was defined as MCI when the actual z-score for a given test was at least 1.5 SD lower than the expected score in at least two tests in one domain or in at least one test in at least two domains.

\section{MRI Data Acquisition}

MRI data were acquired using a Siemens Tim Trio 3T MRI system (Siemens, Erlangen, Germany) by using an 8-channel heal coil. Foam padding and headphones were used to limit head motion and reduce scanner noise. Participants were instructed to keep still and remain motionless. High-resolution structural images were acquired by using 3D T1-weighted magnetizationprepared rapid gradient echo (MPRAGE). Scan parameters were as follows: $\mathrm{TR}=1,900 \mathrm{~ms}, \mathrm{TE}=2.22 \mathrm{~ms}$, flip angle $=9^{\circ}$, matrix size $=256 \times 256,1761 \mathrm{~mm}$ saggital slices. Restingstate pulsed ASL (pASL) perfusion images were collected using Q2TIPS II with the following parameters: labeling time $=700 \mathrm{~ms}$, post-labeling delay $=1,100 \mathrm{~ms}$, slice time $=41 \mathrm{~ms}, \mathrm{TR}=3,000 \mathrm{~ms}$, $\mathrm{TE}=11 \mathrm{~ms}$, field of view $(\mathrm{FOV})=192 \mathrm{~mm} \times 192 \mathrm{~mm}$, matrix $=64 \times 64$, flip angle $=90^{\circ}, 25$ axial slices, and slice thickness $=4 \mathrm{~mm}$. One-hundred and one ASL images were acquired from each subject and the first one was taken as the equilibrium magnetization of the brain (M0) image for CBF calculation. rs-fMRI data were obtained by using an echo-planar imaging sequence that lasted $8 \mathrm{~min}$ (240 volumes) with the following parameters: $\mathrm{TR}=2,000 \mathrm{~ms}$, TE $=30 \mathrm{~ms}$, flip angle $=90^{\circ}, \mathrm{FOV}=240 \mathrm{~mm} \times 240 \mathrm{~mm}$, matrix size $=64 \times 64$, 33 slices, and slice thickness $=4 \mathrm{~mm}$.

\section{Data Preprocessing}

\section{Voxel-Based Morphometry Analysis}

To determine the structural abnormalities in patients, we performed a voxel-based morphometry (VBM) analysis for all structural images of PD-NC, PD-MCI, and HC using SPM12. T1-weighted images were segmented using the unified segmentation model into gray matter (GM), white matter (WM), and cerebrospinal fluid (CSF) based on tissue probability maps (TPMs) in Chinese2020 space $^{1}$ (Liang et al., 2015; Shi et al., 2017). Non-linear warping of GM images was then performed on the GM template in Chinese2020 space. The spatially normalized GM maps were modulated by the Jacobian determinant of the deformation field and corrected for individual brain sizes. The modulated, normalized GM images (voxel size $1 \times 1 \times 1 \mathrm{~mm}^{3}$ ) were smoothed with a 6-mm full width at half maximum (FWHM) isotropic Gaussian kernel.

\section{ASL Data Analysis}

ASL data were preprocessed using $\mathrm{SPM}_{12}{ }^{2}$ based on batch scripts available in ASLtbx (Wang et al., 2008). The following processing was applied: (i) the structural and ASL images were first reoriented to the center of the image matrix; (ii) ASL image pairs were realigned and corrected for head motion (Wang, 2012); (iii) ASL images were then co-registered to their T1 structural images; (iv) high-pass filtering (cutoff $=0.5$ ) was performed for temporal ASL image denoising; (v) Gaussian kernel (FWHM $=6 \mathrm{~mm}$ ) was then applied on the ASL images; (vi) after regressing out residual motions and global signal from ASL images, CBF images were then calculated according to Wang et al. (2005); (vii) T1 images were segmented to GM, WM, and CSF by normalizing into Chinese2020 TMPs and resampled them to $3 \times 3 \times 3 \mathrm{~mm}^{3}$; and (viii) the mean relative $\mathrm{CBF}$ ( $\mathrm{rCBF}$ ) images which were spatially normalized into Chinese2020 template space by using the transformations from segmentation in step (vii) were used to eliminate the global CBF effect.

\section{Rs-fMRI Preprocessing and Functional Connectivity}

Rs-fMRI data were preprocessed using SPM12 and seed-to-voxel correlation analysis was carried out by the FC (CONN) toolbox v17c (Whitfield-Gabrieli and Nieto-Castanon, 2012). The first 10 functional images were discarded to reduce the fluctuation of the MRI signal in the initial stage of scanning. The remaining 230 images of each subject were first corrected for slice timing to reduce the within-scan acquisition time differences between slices and then realigned to eliminate the influence of head motion during the experiment. All subjects included in the present study exhibited head motion less than $1.5 \mathrm{~mm}$ in any of the $x, y$, or $z$ directions and less than $1.5^{\circ}$ of any angular dimension. Next, the realigned images were co-registered to T1 images. Then, T1 images were segmented into GM/WM/CSF by using Chinese2020 TMPs. Then the co-registered images were spatially normalized into Chinese 2020 template space using transformations from segmentation and resampled them to $3 \times 3 \times 3 \mathrm{~mm}^{3}$. Subsequently, the functional images were smoothed with a 6-mm FWHM isotropic Gaussian kernel. After preprocessing, images were then band-pass filtered to $0.008-0.09 \mathrm{~Hz}$ to reduce the influence of noise. Further

\footnotetext{
${ }^{1}$ http://www.chinese-brain-atlases.org

${ }^{2}$ http://www.fil.ion.ucl.ac.uk/spm
} 
denoising steps included regression of the six motion parameters and their first-order derivatives, regression of WM and CSF signals following the implemented CompCor strategy (Behzadi et al., 2007) and a linear detrending. The seed regions were determined by the areas that showed significant $\mathrm{rCBF}$ alterations specific to patients with PD-MCI. The correlation coefficients between the seed voxels and all other brain voxels were computed to generate correlation maps. For group analyses the correlation coefficients were converted to $z$-values using Fisher's $r$-to- $z$ transformation to improve normality (Lowe et al., 1998).

\section{Statistical Analysis}

As to the clinical and neuropsychological measurements, the normality of clinical data and neuropsychological measures was firstly evaluated by a Kolmogorov-Smirnov (KS) test in order to choose parametric tests or non-parametric tests using SPSS 22. Independent one-way analysis of variance (ANOVA; for parametric test) or Kruskal-Wallis testing (for non-parametric test) was performed for comparing the three groups, with Bonferroni correction used for the post hoc comparisons between groups (Student $t$-test for parametric testing and Mann-Whitney for non-parametric testing).

The rCBF maps, seed-voxel FC maps, and GM maps were analyzed using General Linear Model on a voxel-wise comparison across the whole brain. An absolute GM threshold of 0.2 was used to avoid possible edge effects around the border between GM and WM. Alterations in GM volume, $\mathrm{rCBF}$, and seed-to-voxel FC among the three groups were assessed using one-way analysis of covariance (ANCOVA) controlled for nuisance variables. The demographic features that showed significant group differences except for the cognitive profiles were taken as nuisance variables of no interest. Furthermore, the mean frame-wise displacement (FD; Power et al., 2015) and global correlation (GCOR; Saad et al., 2013), which represented the average correlation coefficient between every pair of voxels across the entire brain, were included as nuisance variables to further exclude the influence of head motion and global signal in seed-voxel FC analysis. Post hoc two-sample $t$-tests were then conducted among HC, PD-NC, and PD-MCI masking ANCOVA results inclusively.

GM volume and FC results were reported based on an uncorrected voxel-wise height threshold of $p<0.001$ combined with an FWE-corrected cluster-wise threshold of $p<0.05$. Due to our a priori hypothesis about the localization of perfusion changes, significance level was set at voxel-wise $p<0.001$ uncorrected for CBF analysis. Then a small volume correction (SVC) was applied to investigate which subregions in the posterior parietal and occipital areas might be more affected in PD-MCI patients and results were thresholded at FWE $p<0.05$. Brain region was localized with $\mathrm{xjView}^{3}$.

\section{Correlation Analysis}

In addition, alterations in $\mathrm{rCBF}$ perfusion and FC specific to $\mathrm{PD}$-MCI were defined as regions of interest (ROIs).

\footnotetext{
${ }^{3}$ http://www.alivelearn.net/xjview
}

Partial correlation analyses were further examined between these alterations and neuropsychological performances that exhibited significant differences between PD groups controlled for nuisance variables, similar to the corresponding $\mathrm{CBF}$ or $\mathrm{FC}$ analyses ( $p<0.05$ Bonferroni correction).

\section{RESULTS}

\section{Demographic Results}

As shown in Table 1, ANOVA analyses showed that significant differences were found in education level $(p<0.001)$ and HAMD $(p<0.01)$. Post hoc comparisons revealed that significant lower education level was detected in PD-MCI compared to $\mathrm{HC}$ and $\mathrm{PD}-\mathrm{NC}$, and significant higher HAMD scores were detected in PD-MCI and PD-NC compared to $\mathrm{HC}$. As to the five cognitive domains, individuals with PD-MCI exhibited significant cognitive dysfunction in all five cognitive domains compared to $\mathrm{HC}$ and $\mathrm{PD}-\mathrm{NC}$, with the exception of no significant differences between PD-MCI and PD-NC in verbal fluency tests and AVLT delayed recall, while $\mathrm{PD}-\mathrm{NC}$ patients only exhibited cognitive impairment in the verbal fluency test compared to HC (Table 1). There was no other significant difference in the remaining comparisons.

\section{VBM Results}

Significant GM atrophy was commonly detected in the left caudate (MNI: $-6,7,5$; Chinese2020: $-6,7,4$ with 89 voxels) in patients with $\mathrm{PD}-\mathrm{NC}$ and $\mathrm{PD}-\mathrm{MCI}$ compared to $\mathrm{HC}$. No significant difference was detected between $\mathrm{PD}-\mathrm{NC}$ and PD-MCI.

\section{RCBF Results}

Significant hypoperfusion in the left $\mathrm{PCu}$ was detected in ANCOVA analysis, controlled for education level, and HAMD scores. Post hoc two-sample $t$-testing further revealed that the hypoperfusion in the left $\mathrm{PCu}$ was specific to PD-MCI when compared to PD-NC and HC (Table 2, Figure 1). The region that exhibited hypoperfusion in PD-MCI common to PD-NC was defined as the seed region (MNI: $-14,-68,38$ with 111 voxels; $t=3.96)$ in the FC analysis.

In addition, correlation analysis found that the $\mathrm{rCBF}$ values in the left $\mathrm{PCu}$ region were positively associated with AVLT immediate recall $(r=0.50, p<0.01)$, controlled for education level and HAMD scores (see Figure 1).

\section{FC Results}

Spontaneous brain neural activity that is associated with neurovascular perfusion and hypoperfusion leads to disrupted FC. In the present study, the identified hypoperfusion in the $\mathrm{PCu}$ was further taken as seed regions for FC analysis. The FC results showed that significant decreased FC was detected in the right caudate/putamen in $\mathrm{PD}-\mathrm{MCI}$ when compared to $\mathrm{HC}$ (MNI: $21,18,0$ with 33 voxels; $t=4.96)$ and PD-NC (MNI: $24,12,-3$ with 46 voxels; $t=4.47$; see Table 3, Figure 2). Correlational analysis further revealed that the FC between the seed $\mathrm{PCu}$ and the right caudate/putamen was positively 
TABLE 1 | Demographic and clinical characteristics.

\begin{tabular}{|c|c|c|c|c|}
\hline & HC $(n=25)$ & PD-NC ( $n=27)$ & PD-MCI $(n=27)$ & $p$-value \\
\hline Age (years) ${ }^{\#}$ & $59.44(5.77)$ & $63.11(9.27)$ & $62.59(6.61)$ & 0.17 \\
\hline Gender (male/female) & $11 / 14$ & $15 / 12$ & $16 / 11$ & 0.61 \\
\hline Education (years) & $12.08(2.91)$ & $13.37(2.91)$ & $7.78(4.27)$ & $<0.001^{\dagger \ddagger}$ \\
\hline Disease duration (years) & - & $3.71(2.96)$ & $3.65(2.86)$ & 0.82 \\
\hline Hoehn and Yahr stage & - & $1.88(0.50)$ & $1.71(0.68)$ & 0.20 \\
\hline UPDRS Part II\# & - & $23.82(7.38)$ & $21.38(9.53)$ & 0.38 \\
\hline L-DOPA dose (mg/day) & - & $334.13(255.73)$ & 370.88 (392.0) & 0.93 \\
\hline HAMD & $1.80(2.29)$ & $2.89(1.50)$ & $3.44(2.31)$ & $0.006^{* \dagger}$ \\
\hline \multicolumn{5}{|l|}{ Attention and working memory } \\
\hline Trait making test (B-A) & $30.52(11.97)$ & $37.22(26.09)$ & $99.78(57.97)$ & $<0.001^{\dagger \ddagger}$ \\
\hline Digit span forward & $7.20(1.32)$ & $7.22(1.55)$ & $5.63(1.45)$ & $<0.001^{\dagger \ddagger}$ \\
\hline Digit span backward & $5.44(1.56)$ & $4.63(1.33)$ & $3.52(0.98)$ & $<0.001^{\dagger \ddagger}$ \\
\hline \multicolumn{5}{|l|}{ Executive function } \\
\hline Verbal fluency test & $21.80(4.00)$ & $17.04(3.24)$ & $15.44(4.29)$ & $<0.001^{*} \dagger$ \\
\hline Clock drawing test & $9.52(0.92)$ & $9.52(1.19)$ & $7.07(2.53)$ & $<0.001^{\dagger \ddagger}$ \\
\hline \multicolumn{5}{|l|}{ Language } \\
\hline WAIS-IV & $17.44(2.75)$ & $17.19(3.49)$ & $9.48(3.66)$ & $<0.001^{\dagger \ddagger}$ \\
\hline Boston naming test & $24.76(1.79)$ & 24.07 (2.99) & $19.93(4.44)$ & $<0.001^{\dagger \ddagger}$ \\
\hline \multicolumn{5}{|l|}{ Memory } \\
\hline AVLT immediate recall ${ }^{\#}$ & $29.56(6.64)$ & $26.96(5.91)$ & $21.89(4.60)$ & $<0.001^{\dagger \ddagger}$ \\
\hline AVLT delayed recall & $10.40(2.60)$ & $9.44(2.38)$ & 7.56 (3.70) & $<0.001^{\dagger}$ \\
\hline Logic memory immediate recall & $5.20(1.91)$ & $5.19(1.39)$ & $2.96(1.76)$ & $<0.001^{\dagger \ddagger}$ \\
\hline Logic memory delayed recall & $4.29(1.40)$ & $4.15(1.63)$ & $2.26(1.77)$ & $<0.001^{\dagger \ddagger}$ \\
\hline \multicolumn{5}{|l|}{ Visual spatial function } \\
\hline CLOX & $14.04(0.73)$ & $13.39(1.11)$ & $11.37(2.18)$ & $<0.001^{\dagger \neq}$ \\
\hline JOLO & $21.36(5.15)$ & $19.78(4.79)$ & $14.78(5.61)$ & $<0.001^{\dagger \ddagger}$ \\
\hline
\end{tabular}

Note: data are expressed as mean (standard deviation). Gender data were analyzed with $\chi^{2}$ test. Other p values were derived from Kruskal Wallis test or Mann-Whitney test except for \# that was derived from the independent one-way analysis of variance (ANOVA) or two sample t-test. * Post hoc comparisons showed significant differences between healthy controls (HCs) and patients with Parkinson's disease with normal cognition (PD-NC); ${ }^{\dagger}$ post hoc comparisons showed significant differences between HCs and patients with PD-mild cognitive impairment (PD-MCl); ${ }^{\ddagger}$ post hoc comparisons showed significant differences between patients with PD-NC and those with PD-MCl. UPDRS, unified Parkinson's disease rating scale; HAMD, Hamilton depression rating scale; JOLO, Benton Judgment of Line Orientation.

TABLE 2 | Relative CBF ( $\mathrm{rCBF}$ ) changes among groups of HC, PD-NC, and PD-MCl.

\begin{tabular}{lcccc}
\hline Region & $\begin{array}{c}\text { Cluster size } \\
\text { (voxel) }\end{array}$ & $\begin{array}{c}\text { MNI } \\
(\boldsymbol{x}, \boldsymbol{y}, \mathbf{z})\end{array}$ & $\begin{array}{c}\text { Chinese2020 } \\
(\boldsymbol{x}, \boldsymbol{y}, \mathbf{z})\end{array}$ & $\boldsymbol{t}$-value \\
\hline $\mathrm{HC}>\mathrm{PD}-\mathrm{MCl}$ & 118 & $(-14,-68,38)$ & $(-14,-59,39)$ & 3.96 \\
$\mathrm{Lt.PCu}$ & 194 & $(-15,-69,36)$ & $(-15,-60,38)$ & 4.30 \\
$\mathrm{PD}-\mathrm{NC}>\mathrm{PD}-\mathrm{MCl}$ & & & \\
Lt.PCu & 194
\end{tabular}

Note: PCu, precuneus; Lt, left.

correlated with WMS logical memory immediate recall $(r=0.49$, $p<0.01)$ and delayed recall $(r=0.63, p<0.001)$ performances controlled for the education level, and HAMD scores (see Figure 2).

\section{DISCUSSION}

The current study investigated the specific alteration pattern of $\mathrm{CBF}$ perfusion in PD-MCI in comparison to both PD-NC and $\mathrm{HC}$, and further investigated how neurovascular dysfunction affects neural activity in PDMCI. Specifically, the PCu hypoperfusion was identified in PD-MCI, and the reduced PCu-FC in the striatum was further detected specific to this disease. Moreover, the $\mathrm{PCu}$ dysfunctions (i.e., hypoperfusion and disrupted FC) were positively correlated with memory performance in PD-MCI, which suggests that malfunction (neurovascular and neural functional activity) in the posteromedial parietal region

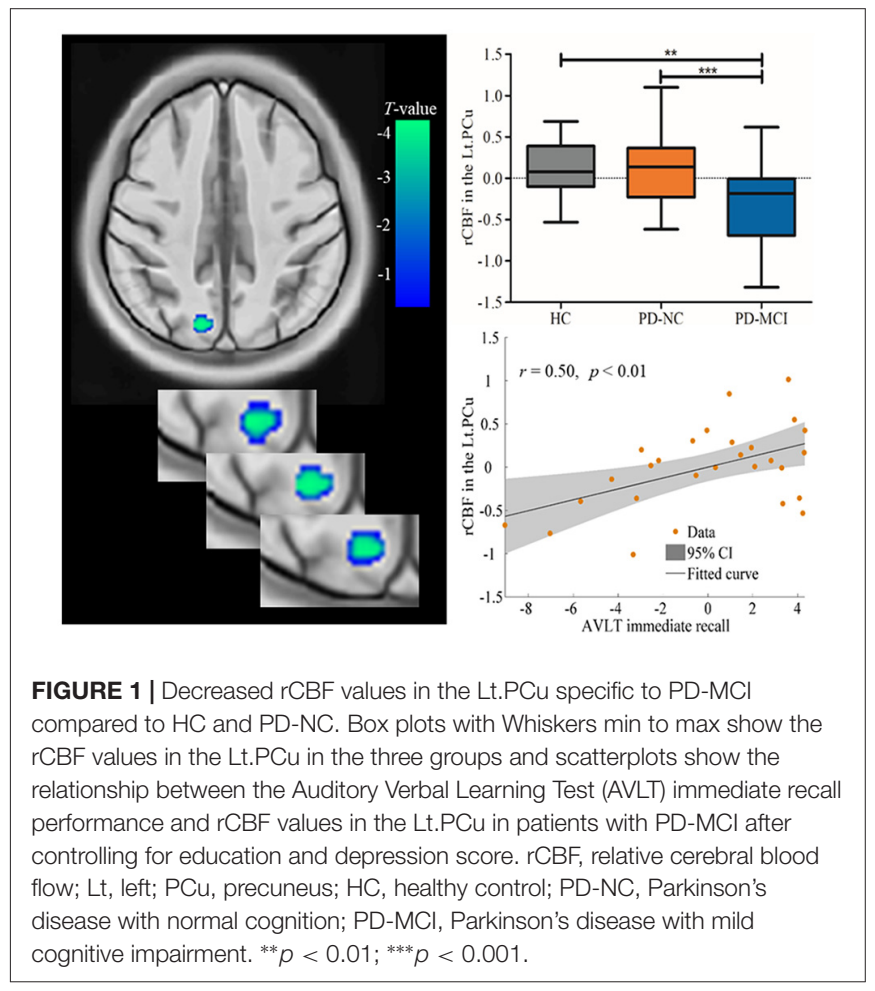

may contribute to memory decline within individuals with PD-MCI. 
TABLE 3 | Decreased functional connectivity (FC) in seed region of the Lt.PCu in $\mathrm{PD}-\mathrm{MCl}$ compared to $\mathrm{HC}$ and PD-NC.

\begin{tabular}{lcccc}
\hline Region & $\begin{array}{c}\text { Cluster size } \\
\text { (voxel) }\end{array}$ & $\begin{array}{c}\text { MNI } \\
(\boldsymbol{x}, \boldsymbol{y}, \mathbf{z})\end{array}$ & $\begin{array}{c}\text { Chinese2020 } \\
(\boldsymbol{x}, \boldsymbol{y}, \mathbf{z})\end{array}$ & $\boldsymbol{T}$-value \\
\hline $\begin{array}{l}\mathrm{HC}>\mathrm{PD}-\mathrm{MCl} \\
\text { Rt.Caudate/Putamen }\end{array}$ & 33 & $(21,18,0)$ & $(20,16,-1)$ & 4.96 \\
$\begin{array}{l}\text { PD-NC }>\text { PD-MCl } \\
\text { Rt.Caudate/Putamen }\end{array}$ & 46 & $(24,12,-3)$ & $(23,11,-3)$ & 4.47 \\
\hline
\end{tabular}

Note: Rt, right

\section{GM Atrophy in PD}

In line with previous PD studies (Brenneis et al., 2003; Lee et al., 2011), in the present study significant GM atrophy was detected in the left caudate in PD patients (independently of the cognitive status). Previous studies have reported GM atrophy in PD-MCI but still lack consistency. For example, Chen et al. (2017) reported cortical atrophy in frontal and temporal areas in PD-MCI, but Hattori et al. (2012) failed to detect any difference between PD-MCI and PD-NC or HC. These inconsistences might be due to the heterogeneities of patients, such as different stages of disease and medicationrelated confounding.

\section{Hypoperfusion in the PCu Specific to PD-MCI}

In line with a previous SPECT study (Nobili et al., 2009), posterior cortical hypoperfusion, particularly in the $\mathrm{PCu}$, was found specific to PD-MCI. Recently, studies have indicated that the $\mathrm{PCu}$ is a key node of the "parietal memory network" $(\mathrm{PMN})$, which is recruited in memory encoding and retrieval tasks (Gilmore et al., 2015; Hu et al., 2016). Furthermore, in the current study, it was revealed that the PCu perfusion positively correlated to memory function measured by AVLT immediate recall, which implies the neural characteristics of memory deficits in PD-MCI. To date, no ASL study has characterized the CBF perfusion pattern specific to well-defined PD-MCI patients in comparison to PD-NC and HC. Recently, the only ASL study including PD-MCI found decreased perfusion in individuals with $\mathrm{PD}$ in full range of cognitive status compared to controls, whereas specific perfusion characteristics in PD-MCI were not specified
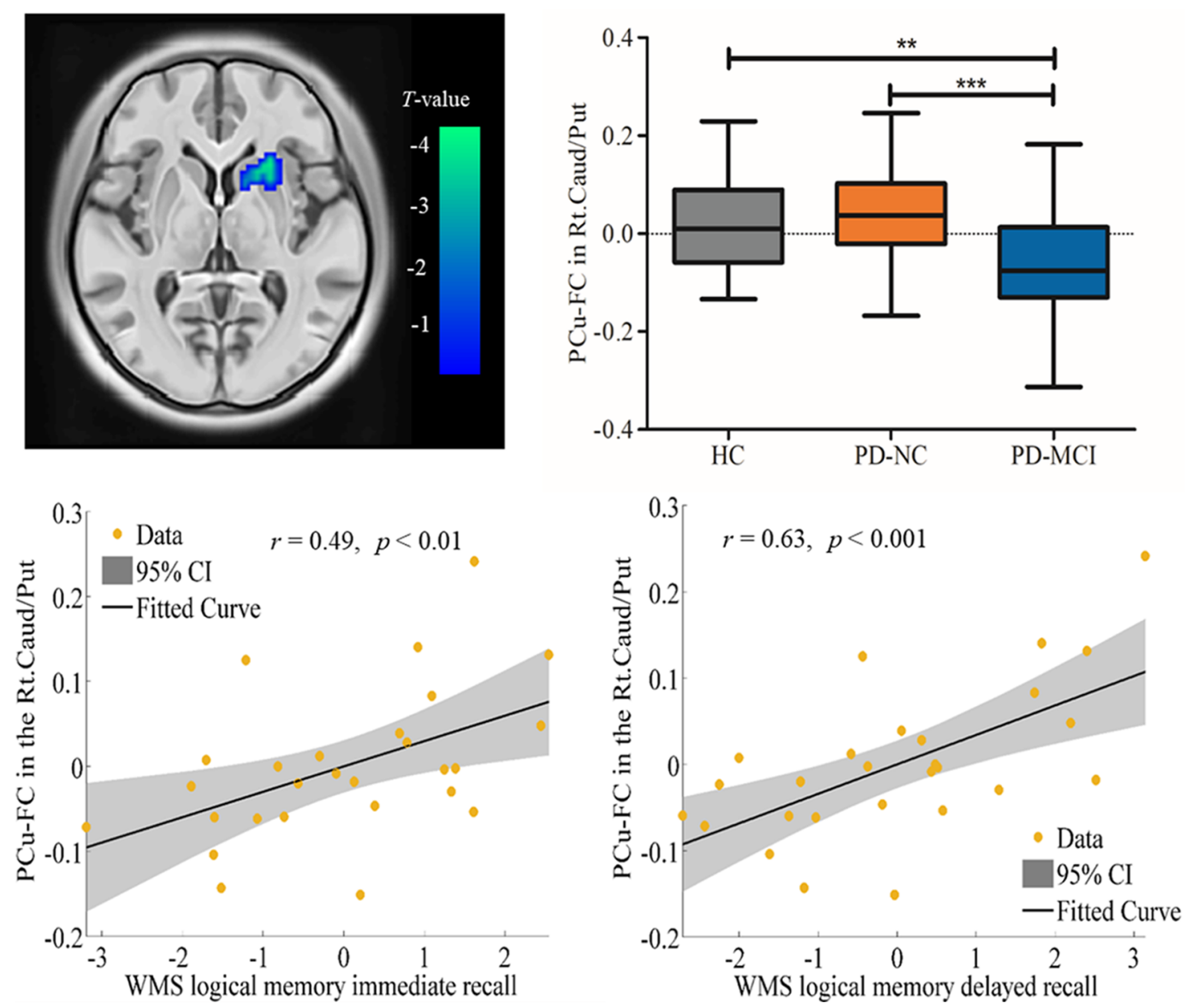

FIGURE 2 | Decreased functional connectivity (FC) in the seed region of the Lt.PCu in the Rt.Caud/Put specific to PD-MCl compared to HC and PD-NC. Box plots with whiskers min to max show the FC values between the Lt.PCu and the Rt.Caud/Put in the three groups and scatterplots show the relationship between the FC strength in the two regions and WMS logical memory performances in patients with PD-MCl after controlling for education and depression score. Lt, left; Rt, right; PCu, precuneus; Caud, caudate; Put, putamen; HC, healthy control; PD-NC, Parkinson's disease with normal cognition; PD-MCl, Parkinson's disease with mild cognitive impairment. ${ }^{* *} p<0.01 ;{ }^{* * *} p<0.001$. 
as no post hoc comparison was performed after ANOVA (Melzer et al., 2011). In addition, previous morphometry studies have revealed GM atrophy in PD patients even at early stage (Hong et al., 2012; Lee et al., 2013; Jia et al., 2015), which may interact with cortical perfusion deficits (Fernández-Seara et al., 2012).

More recently, a study in individuals with non-demented PD (combined PD-NC and PD-MCI) found that CBF abnormalities in the $\mathrm{PCu}$ are associated with global cognitive deficits (Syrimi et al., 2017). However, the present study did not detect significant differences between PD-NC and HC. The following explanations may account for the inconsistency. One possibility was that in the present study the cognitive status in $\mathrm{PD}$ patients was comprehensively examined including five cognitive domains and was well-matched between PD-NC and $\mathrm{HC}$; while in the other study cognitive status was tested by a global cognitive assessment, therefore it lacked a detailed neuropsychological examination in non-demented PD. In any case, the cognitive status in controls was not available. Furthermore, the PD patients in the present study were examined in their OFF medication state, while in that study PD participants were tested in their ON medication state, which could induce CBF changes in PD (Kobari et al., 1995). Taken together, the current findings suggested that $\mathrm{PCu}$ hypoperfusion might be a specific characteristic of PD$\mathrm{MCI}$, which further differentiates the neural underpinnings of cognitive decline in PD-MCI from non-demented PD.

\section{Decreased PCu-FC in the Striatum}

Neurovascular function is tightly coupled with neural activity measured by BOLD signal. In the present study, the reduced $\mathrm{PCu} \mathrm{FC}$ in the hypoperfusion region with the striatum (caudate/putamen) was detected in PD-MCI, controlling for the potential effect of brain structural alteration. Indeed, the striatum and PCu have been reported to be anatomically and functionally connected (Selemon and Goldman-Rakic, 1985; Cavanna and Trimble, 2006), which implies the abnormal perfusion in the $\mathrm{PCu}$ in $\mathrm{PD}-\mathrm{MCI}$ afflicts its functional synchronization with the striatum. It is noteworthy that the loss of dopaminergic neurons in the nigrostriatal pathway results in the depletion of dopamine in the striatum (Ravina et al., 2012; Buddhala et al., 2015). Specifically, alteration of the striatum in PD has been consistently reported in non-demented PD both structurally (Kostic et al., 2012; Jia et al., 2015) and functionally (Shin et al.,

\section{REFERENCES}

Aarsland, D., Bronnick, K., Larsen, J. P., Tysnes, O. B., Alves, G., and Norwegian ParkWest Study Group. (2009). Cognitive impairment in incident, untreated Parkinson disease: the norwegian parkwest study. Neurology 72, 1121-1126. doi: 10.1212/01.wnl.0000338632.00552.cb

AI-Bachari, S., Parkes, L. M., Vidyasagar, R., Hanby, M. F., Tharaken, V., Leroi, I., et al. (2014). Arterial spin labelling reveals prolonged arterial arrival time in idiopathic Parkinson's disease. Neuroimage Clin. 6, 1-8. doi: 10.1016/j.nicl. 2014.07.014

Amboni, M., Tessitore, A., Esposito, F., Santangelo, G., Picillo, M., Vitale, C., et al. (2015). Resting-state functional connectivity associated with mild cognitive impairment in Parkinson's disease. J. Neurol. 262, 425-434. doi: 10.1007/s00415-014-7591-5

Baggio, H. C., Segura, B., Sala-LIonch, R., Marti, M. J., Valldeoriola, F., Compta, Y., et al. (2015). Cognitive impairment and resting-state network connectivity
2016). In addition, a previous study has indicated that older adults recruit the opposing homogenous hemispheric regions to maintain task performance (Cabeza, 2002). In the present study, the decreased $\mathrm{PCu}-\mathrm{FC}$ in the contralateral striatum in patients with PD-MCI may imply a damaged ability to recruit interhemispheric FC for compensation for age-related declines in the structural integrity of long intrahemispheric connections (Hermundstad et al., 2013). The positive association between the $\mathrm{PCu}-\mathrm{FC}$ in the striatum and memory performance measured by logic memory in PD-MCI, further supports the predictive potential of $\mathrm{PCu}$ degeneration on memory dysfunction in this disease.

However, a limitation should be recognized in the current study. Besides ASL, WM status could also reflect neurovascular integrity. A recent study has reported significant DTI changes in PD that precede GM changes (Rektor et al., 2018). Further studies need to explore neurovascular integrity considering the WM alteration revealed by DTI data. Overall, the current findings of $\mathrm{PCu}$ abnormality (i.e., hypoperfusion and abnormal FC) specific to PD-MCI, suggest that combination between CBF and BOLD could provide insight into the mechanisms underlying cognitive decline in PD-MCI.

\section{AUTHOR CONTRIBUTIONS}

$\mathrm{KL}, \mathrm{PL}$, and XF contributed to the study conception and design. YL carried out data collection. Data were analyzed and interpreted by XJ. The drafting of manuscript was written by XJ and was critically revised by PL and XF. The final approval of the version to be published was confirmed by PL, XF and XJ.

\section{FUNDING}

This work was supported by grants from the National Natural Science Foundation of China $(61473196,61621136008$, and 61672065), Beijing Talents Foundation (2016000021223TD07), China Postdoctoral Science Foundation (2015M570165), Beijing Municipal Administration of Hospitals Clinical Medicine Development of Special Funding (ZYLX201609), and Key Projects in the National Science and Technology Pillar Program during the Twelfth Five-year Plan Period (2012BAI10B04).

in Parkinson's disease. Hum. Brain Mapp. 36, 199-212. doi: 10.1002/hbm. 22622

Behzadi, Y., Restom, K., Liau, J., and Liu, T. T. (2007). A component based noise correction method (CompCor) for BOLD and perfusion based fMRI Neuroimage 37, 90-101. doi: 10.1016/j.neuroimage.2007.04.042

Bezdicek, O., Ballarini, T., Ruzicka, F., Roth, J., Mueller, K., Jech, R., et al (2018). Mild cognitive impairment disrupts attention network connectivity in Parkinson's disease: a combined multimodal MRI and meta-analytical study. Neuropsychologia 12, 105-115. doi: 10.1016/j.neuropsychologia.2018. 03.011

Brenneis, C., Seppi, K., Schocke, M. F., Muller, J., Luginger, E., Bosch, S., et al. (2003). Voxel-based morphometry detects cortical atrophy in the Parkinson variant of multiple system atrophy. Mov. Disord. 18, 1132-1138. doi: 10.1002/mds.10502

Buddhala, C., Loftin, S. K., Kuley, B. M., Cairns, N. J., Campbell, M. C., Perlmutter, J. S., et al. (2015). Dopaminergic, serotonergic, and noradrenergic 
deficits in Parkinson disease. Ann. Clin. Transl. Neurol. 2, 949-959. doi: $10.1002 / \mathrm{acn} 3.246$

Cabeza, R. (2002). Hemispheric asymmetry reduction in older adults: the HAROLD model. Psychol. Aging 17, 85-100. doi: 10.1037//0882-7974.17.1.85

Cavanna, A. E., and Trimble, M. R. (2006). The precuneus: a review of its functional anatomy and behavioural correlates. Brain 129, 564-583. doi: 10.1093/brain/awl004

Chen, B., Wang, S., Sun, W., Shang, X., Liu, H., Liu, G., et al. (2017). Functional and structural changes in gray matter of Parkinson's disease patients with mild cognitive impairment. Eur. J. Radiol. 93, 16-23. doi: 10.1016/j.ejrad.2017. 05.018

D'Esposito, M., Deouell, L. Y., and Gazzaley, A. (2003). Alterations in the bold FMRI signal with ageing and disease: a challenge for neuroimaging. Nat. Rev. Neurosci. 4, 863-872. doi: 10.1038/nrn1246

Detre, J. A., Leigh, J. S., Williams, D. S., and Koretsky, A. P. (1992). Perfusion imaging. Magn. Reson. Med. 23, 37-45. doi: 10.1002/mrm.19102 30106

Fernández-Seara, M. A., Mengual, E., Vidorreta, M., Aznárez-Sanado, M., Loayza, F. R., Villagra, F., et al. (2012). Cortical hypoperfusion in Parkinson's disease assessed using arterial spin labeled perfusion MRI. Neuroimage 59, 2743-2750. doi: 10.1016/j.neuroimage.2011.10.033

Gilmore, A. W., Nelson, S. M., and McDermott, K. B. (2015). A parietal memory network revealed by multiple MRI methods. Trends Cogn. Sci. 19, 534-543. doi: 10.1016/j.tics.2015.07.004

Hamilton, M. (1960). A rating scale for depression. J. Neurol. Neurosurg. Psychiatry 23, 56-62. doi: 10.1136/jnnp.23.1.56

Hattori, T., Orimo, S., Aoki, S., Ito, K., Abe, O., Amano, A., et al. (2012). Cognitive status correlates with white matter alteration in Parkinson's disease. Hum. Brain Mapp. 33, 727-739. doi: 10.1002/hbm.21245

Hermundstad, A. M., Bassett, D. S., Brown, K. S., Aminoff, E. M., Clewett, D., Freeman, S., et al. (2013). Structural foundations of resting-state and task-based functional connectivity in the human brain. Proc. Natl. Acad. Sci. U S A 110, 6169-6174. doi: 10.1073/pnas.1219562110

Hong, J., Lee, J. E., Sohn, Y. H., and Lee, P. H. (2012). Neurocognitive and atrophic patterns in Parkinson's disease based on subjective memory complaints. J. Neurol. 259, 1706-1712. doi: 10.1007/s00415-011-6404-3

Hosokai, Y., Nishio, Y., Hirayama, K., Takeda, A., Ishioka, T., Sawada, Y., et al. (2009). Distinct patterns of regional cerebral glucose metabolism in Parkinson's disease with and without mild cognitive impairment. Mov. Disord. 24, 854-862. doi: 10.1002/mds.22444

Hu, Y., Wang, J., Li, C., Wang, Y., Yang, Z., and Zuo, X. (2016). Segregation between the parietal memory network and the default model network: effects of spatial smoothing and model order in ICA. Sci. Bull. 61, 1844-1854. doi: 10.1007/s11434-016-1202-Z

Hughes, A. J., Daniel, S. E., Kilford, L., and Lees, A. J. (1992). Accuracy of clinical diagnosis of idiopathic Parkinson's disease: a clinico-pathological study of 100 cases. J. Neurol. Neurosurg. Psychiatry 55, 181-184. doi: 10.1136/jnnp.55. 3.181

Janvin, C. C., Larsen, J. P., Aarsland, D., and Hugdahl, K. (2006). Subtypes of mild cognitive impairment in Parkinson's disease: progression to dementia. Mov. Disord. 21, 1343-1349. doi: 10.1002/mds.20974

Jia, X., Liang, P., Li, Y., Shi, L., Wang, D., and Li, K. (2015). A longitudinal study of gray matter changes in Parkinson's disease. AJNR Am. J. Neuroradiol. 36, 2219-2226. doi: 10.3174/ajnr.A4447

Kehagia, A. A., Barker, R. A., and Robbins, T. W. (2010). Neuropsychological and clinical heterogeneity of cognitive impairment and dementia in patients with Parkinson's disease. Lancet Neurol. 9, 1200-1213. doi: 10.1016/S14744422(10)70212-X

Kobari, M., Fukuuchi, Y., Shinohara, T., Obara, K., and Nogawa, S. (1995). Levodopa-induced local cerebral blood flow changes in Parkinson's disease and related disorders. J. Neurol. Sci. 128, 212-218. doi: 10.1016/0022510x(94)00237-i

Kostic, V. S., Agosta, F., Pievani, M., Stefanova, E., Jecmenica-Lukic, M., Scarale, A., et al. (2012). Pattern of brain tissue loss associated with freezing of gait in Parkinson disease. Neurology 78, 409-416. doi: 10.1212/WNL. 0b013e318245d23c

Lee, E. Y., Sen, S., Eslinger, P. J., Wagner, D., Shaffer, M. L., Kong, L., et al. (2013). Early cortical gray matter loss and cognitive correlates in non-demented
Parkinson's patients. Parkinsonism Relat. Disord. 19, 1088-1093. doi: 10.1016/j. parkreldis.2013.07.018

Lee, S. H., Kim, S. S., Tae, W. S., Lee, S. Y., Choi, J. W., Koh, S. B., et al. (2011) Regional volume analysis of the Parkinson disease brain in early disease stage: gray matter, white matter, striatum and thalamus. AJNR Am. J. Neuroradiol. 32, 682-687. doi: 10.3174/ajnr.A2372

Liang, P., Shi, L., Chen, N., Luo, Y., Wang, X., Liu, K., et al. (2015). Construction of brain atlases based on a multi-center MRI dataset of 2020 Chinese adults. Sci. Rep. 5:18216. doi: 10.1038/srep18216

Litvan, I., Goldman, J. G., Troster, A. I., Schmand, B. A., Weintraub, D., Petersen, R. C., et al. (2012). Diagnostic criteria for mild cognitive impairment in Parkinson's disease: movement disorder society task force guidelines. Mov. Disord. 27, 349-356. doi: 10.1002/mds.24893

Lowe, M. J., Mock, B. J., and Sorenson, J. A. (1998). Functional connectivity in single and multislice echoplanar imaging using resting-state fluctuations. Neuroimage 7, 119-132. doi: 10.1006/nimg.1997.0315

Ma, Y., Tang, C., Spetsieris, P. G., Dhawan, V., and Eidelberg, D. (2007). Abnormal metabolic network activity in Parkinson's disease: test-retest reproducibility. J. Cereb. Blood Flow Metab. 27, 597-605. doi: 10.1038/sj.jcbfm.9600358

Ma, Y., Huang, C., Dyke, J. P., Pan, H., Alsop, D., Feigin, A., et al. (2010). Parkinson's disease spatial covariance pattern: noninvasive quantification with perfusion MRI. J. Cereb. Blood Flow Metab 30, 505-509. doi: 10.1038/jcbfm. 2009.256

Melzer, T. R., Watts, R., MacAskill, M. R., Pearson, J. F., Rueger, S., Pitcher, T. L., et al. (2011). Arterial spin labelling reveals an abnormal cerebral perfusion pattern in Parkinson's disease. Brain 134, 845-855. doi: 10.1093/brain/ awq377

Musiek, E. S., Chen, Y., Korczykowski, M., Saboury, B., Martinez, P. M., Reddin, J. S., et al. (2012). Direct comparison of fluorodeoxyglucose positron emission tomography and arterial spin labeling magnetic resonance imaging in Alzheimer's disease. Alzheimer's Dement. 8, 51-59. doi: 10.1016/j.jalz.2011. 06.003

Muslimovic, D., Post, B., Speelman, J. D., and Schmand, B. (2005). Cognitive profile of patients with newly diagnosed Parkinson disease. Neurology 65, 1239-1245. doi: 10.1212/01.wnl.0000180516.69442.95

Nobili, F., Abbruzzese, G., Morbelli, S., Marchese, R., Girtler, N., Dessi, B., et al. (2009). Amnestic mild cognitive impairment in Parkinson's disease: a brain perfusion SPECT study. Mov. Disord. 24, 414-421. doi: 10.1002/mds. 22381

Power, J. D., Schlaggar, B. L., and Petersen, S. E. (2015). Recent progress and outstanding issues in motion correction in resting state fMRI. Neuroimage 105, 536-551. doi: 10.1016/j.neuroimage.2014.10.044

Ravina, B., Marek, K., Eberly, S., Oakes, D., Kurlan, R., Ascherio, A., et al. (2012). Dopamine transporter imaging is associated with long-term outcomes in Parkinson's disease. Mov. Disord. 27, 1392-1397. doi: 10.1002/mds. 25157

Rektor, I., Svátkova, A., Vojtíšek, L., Zikmundová, I., Vaníček, J., Király, A., et al. (2018). White matter alterations in Parkinson's disease with normal cognition precede grey matter atrophy. PLOS One 13:e0187939. doi: 10.1371/journal. pone.0187939

Saad, Z. S., Reynolds, R. C., Jo, H. J., Gotts, S. J., Chen, G., Martin, A., et al. (2013). Correcting brain-wide correlation differences in resting-state FMRI. Brain Connect. 3, 339-352. doi: 10.1089/brain.2013.0156

Selemon, L. D., and Goldman-Rakic, P. S. (1985). Longitudinal topography and interdigitation of corticostriatal projections in the rhesus monkey. J. Neurosci. 5, 776-794. doi: 10.1523/jneurosci.05-03-00776.1985

Shi, L., Liang, P., Luo, Y., Liu, K., Mok, V. C. T., Chu, W. C. W., et al. (2017). Using large-scale statistical Chinese brain template (Chinese2020) in popular neuroimage analysis toolkits. Front. Hum. Neurosci. 11:414. doi: 10.3389/fnhum.2017.00414

Shin, N. Y., Shin, Y. S., Lee, P. H., Yoon, U., Han, S., Kim, D. J., et al. (2016). Different functional and microstructural changes depending on duration of mild cognitive impairment in Parkinson disease. AJNR Am. J. Neuroradiol. 37, 897-903. doi: 10.3174/ajnr.A4626

Syrimi, Z. J., Vojtisek, L., Eliasova, I., Viskova, J., Svatkova, A., Vanicek, J., et al. (2017). Arterial spin labelling detects posterior cortical hypoperfusion in non-demented patients with Parkinson's disease. J. Neural Transm. 124, 551-557. doi: 10.1007/s00702-017-1703-1 
Teune, L. K., Renken, R. J., and de Jong, B. M. (2014). Parkinson's disease related perfusion and glucose metabolic brain patterns identified with PCASL-MRI and FDG-PET imaging. Neuroimage Clin. 5, 240-244. doi: 10.1016/j.nicl.2014. 06.007

Wang, J., Zhang, Y., Wolf, R. L., Roc, A. C., Alsop, D. C., and Detre, J. A. (2005). Amplitude-modulated continuous arterial spin-labeling 3.0-T perfusion $\mathrm{MR}$ imaging with a single coil: feasibility study. Radiology 235, 218-228. doi: $10.1148 /$ radiol.2351031663

Wang, Z. (2012). Improving cerebral blood flow quantification for arterial spin labeled perfusion MRI by removing residual motion artifacts and global signal fluctuations. Magn. Reson. Imaging 30, 1409-1415. doi: 10.1016/j.mri.2012. 05.004

Wang, Z., Aguirre, G. K., Rao, H., Wang, J., Fernandez-Seara, M. A., Childress, A. R., et al. (2008). Empirical optimization of ASL data analysis using an ASL data processing toolbox: ASLtbx. Magn. Reson. Imaging 26, 261-269. doi: 10.1016/j.mri.2007.07.003

Whitfield-Gabrieli, S., and Nieto-Castanon, A. (2012). Conn: a functional connectivity toolbox for correlated and anticorrelated brain networks. Brain Connect. 2, 125-141. doi: 10.1089/brain.2012.0073

Williams, D. S., Detre, J. A., Leigh, J. S., and Koretsky, A. P. (1992). Magnetic resonance imaging of perfusion using spin inversion of arterial water. Proc. Natl. Acad. Sci. U S A 89, 212-216. doi: 10.1073/pnas.89.9. $4220 \mathrm{e}$

Wolf, R. L., and Detre, J. A. (2007). Clinical neuroimaging using arterial spin labeled perfusion magnetic resonance imaging. Neurotherapeutics 4, 346-359. doi: 10.1016/j.nurt.2007.04.005

Yoshiura, T., Hiwatashi, A., Noguchi, T., Yamashita, K., Ohyagi, Y., Monji, A., et al. (2009). Arterial spin labelling at 3-T MR imaging for detection of individuals with Alzheimer's disease. Eur. Radiol. 19, 2819-2825. doi: 10.1007/s00330-009-1511-6

Conflict of Interest Statement: The authors declare that the research was conducted in the absence of any commercial or financial relationships that could be construed as a potential conflict of interest.

Copyright (C) $2019 \mathrm{Jia}, \mathrm{Li}, \mathrm{Li}$, Liang and Fu. This is an open-access article distributed under the terms of the Creative Commons Attribution License (CC BY). The use, distribution or reproduction in other forums is permitted, provided the original author(s) and the copyright owner(s) are credited and that the original publication in this journal is cited, in accordance with accepted academic practice. No use, distribution or reproduction is permitted which does not comply with these terms. 